\title{
Review Article \\ Role of Cancer-Associated Fibroblast in Gastric Cancer Progression and Resistance to Treatments
}

\author{
In-Hye Ham, ${ }^{1}$ Dagyeong Lee, ${ }^{1,2,3}$ and Hoon Hur $\mathbb{D}^{1,2,3}$ \\ ${ }^{1}$ Department of Surgery, Ajou University School of Medicine, Suwon, Republic of Korea \\ ${ }^{2}$ Brain Korea 21 Plus Research Center for Biomedical Sciences, Ajou University, Suwon, Republic of Korea \\ ${ }^{3}$ Department of Biomedical Science, Graduated School of Ajou University, Suwon, Republic of Korea
}

Correspondence should be addressed to Hoon Hur; hhcmc75@ajou.ac.kr

Received 28 February 2019; Accepted 23 May 2019; Published 9 June 2019

Guest Editor: Yang Ge

Copyright (C) 2019 In-Hye Ham et al. This is an open access article distributed under the Creative Commons Attribution License, which permits unrestricted use, distribution, and reproduction in any medium, provided the original work is properly cited.

\begin{abstract}
Although the survival of gastric cancer (GC) patients has gradually improved, the outcomes of advanced GC patients remain unsatisfactory despite standard treatment with conventional chemotherapy or targeted agents. Several studies have shown that cancer-associated fibroblasts (CAFs), a major component of tumor stroma in GC, may have significant roles in GC progression and resistance to treatments. CAFs are a major source of various secreted molecules in the tumor microenvironment, which stimulate cancer cells and other noncancerous components of GC. Surprisingly, these factors could be involved in gastric carcinogenesis. Cytokines, including interleukin-6 and interleukin-11, or growth factors, such as fibroblast growth factor produced from CAFs, can directly activate GC cells and consequently lead to the development of an aggressive phenotype. Galectin-1 or hepatocyte growth factor can be involved in CAF-derived neovascularization in GC. In addition, recent studies showed that CAFs can affect tumor immunity through M2 polarization of tumor-associated macrophages. Finally, the current study aimed to introduce several inhibitory agents and evaluate their suppressive effects on CAFs in patients with GC progression. However, further studies are required to evaluate their safety and select appropriate patients for application in clinical settings.
\end{abstract}

\section{Introduction}

Gastric cancer (GC) is one of the most common malignancies worldwide and is a major cause of cancer-related mortality [1]. The standard treatment for GC without distant metastasis is resection of the stomach and proper lymph node dissection, and postoperative systemic chemotherapies are recommended for stage II or III $[2,3]$. Despite standard treatment for patients with resectable GC, patients with advanced stage GC still show poor prognosis; therefore, the 5 -year overall survival rate of patients with stage III was about 20\%-40\% [4-6]. Meanwhile, the therapeutic option for GC patients with distant metastasis (stage IV) or patients with recurrence after resection is systemic chemotherapy with multidrug regimens, but the outcomes are poor with a reported mean survival time of about 10 months or less [7-10]. Recently, a variety of molecularly targeted agents has been proposed to enhance the survival rate. However, most clinical trials either have not shown a survival benefit, except for trastuzumab and ramucirumab as combined agents with palliative chemotherapy, or are still ongoing [11]. The limited benefit of treatments for GC is increasingly attributed to the tumor stroma, including extracellular matrix (ECM), fibroblasts, immune cells, and microvasculature, as it is well known that GC has a profuse, noncancerous proportion that contributes to GC progression [12-14].

Among these various components in the tumor stroma, cancer-associated fibroblasts (CAFs) have been suggested to play a key role in tumor development [15]. CAFs remarkably influence the tumor microenvironment (TME) via the secretion of cytokines, chemokines, and growth factors [16, 17]. Those secreted proteins enhance cellular migration, alter the metabolism of epithelial tumor cells $[18,19]$, control the metabolic flexibility of cancer cells [20], and play a significant 
role in the development of resistance to therapeutic agents $[21,22]$. The function of CAFs in cancer progression has been explored in various in vitro experimental models using CAFs isolated from primary human solid tumor tissues [23]. Usually, the cancer cells were cocultured with CAFs, which have shown increased migration, invasion, and survival ability, and tumorigenesis of cancer cells was compared with those cocultured with normal fibroblasts [23-25]. Not only in vitro but also in vivo experiments have confirmed that CAFs advance tumor growth and promote metastasis of cancer cells when coinjected into murine xenograft models $[23,26]$. In spite of these results, the unique makers of CAFs and main contributors according to the type of cancer have not been clarified. There have been several well-established indicators of CAFs, such as platelet-derived growth factor $\alpha$ (PDGRF $\alpha), \operatorname{PDGFR} \beta$, and alpha-smooth muscle actin ( $\alpha$ SMA) [15]. However, these markers are typically expressed only in a fraction of fibroblasts within the tumor and are not specific to CAFs. In addition, CAF-derived contributors involved in cancer progression could differ depending on the type of cancer, because they have different carcinogenesis and progression mechanism. Therefore, the mechanisms of communication between CAFs and specific types of cancers need to be investigated.

Based on those characteristics of CAFs in solid cancers, previous studies have shown that the histologic accumulation of CAF in various types of cancers (colon, esophagus, breast, and liver cancer) could be a poor prognostic maker [27-30]. In GC, type IV of traditional Borrmann's classification has a profuse fibrotic stroma showing poor prognosis due to high recurrence in the peritoneum [31-33]. Those clinical studies have implied that CAFs accumulated in GC tissues might enhance the progression and metastasis of GC. In addition, the high throughput gene expression profiling in GC tissues revealed that the tumors with a high expression of "stroma signature" genes contained a high proportion of fibrotic stroma including CAFs and could be a surrogate marker for predicting the prognosis of GC patients [34]. Our previous study also investigated if the accumulation of fibroblast in a specific subtype of GC, signet ring cell carcinoma, was related to the clinical outcomes [35]. In this study, a higher proportion of CAFs, which was evaluated by immunohistochemical staining for $\alpha$-smooth muscle actin and Masson's trichrome staining for stromal collagen, was significantly related to poor prognosis than a lower proportion of CAFs. Taken together, it would be easily assumed that CAFs have a big impact on the GC progression, due to their direct effects on cancer cells or indirect effects on other ecosystems within malignant tumors. The former could induce the stemness or metastatic potency of cancer cells through paracrine or direct contact, while the latter could control noncancerous microenvironments such as angiogenesis or tumor immunity.

To explore the function of CAFs in malignant tumors through experimental models, CAF should be steadily isolated from bulky tumors. To date, several methods to isolate fibroblasts from GC tissues have been established and isolated fibroblasts were confirmed by expression of specific markers [35-38]. For example, the fresh tissues harvested from patients were immediately moved into a clean bench and were cut into small pieces in a culture dish. After mincing with scalpels, a coverslip was placed over the tissue forming a sandwich. Fibroblasts usually outgrew in a monolayer and were subsequently collected [35]. However, because those cells were not immortalized, most researchers used the fibroblasts with low passage number for subsequent experiments.

We aimed to provide an update on the mechanism of CAF-induced GC progression in the view of tumorigenesis, invasion and metastasis, angiogenesis, and tumor immunity. We also aimed to introduce the potential therapeutic strategies that can target the effect of CAFs on the GC cells.

\section{Origin of CAFs Accumulated in GC}

Although CAFs are the predominant cell type within the tumor stroma of various solid cancers, the origin of CAFs is not fully understood. Previous studies have suggested some candidates for the origin of CAFs such as fibroblast in normal tissues [63], specific cells around vessels such as pericytes and vascular smooth muscle cells [64], endothelial cells [65], and bone marrow-derived stem cells [66]. In GC, a pericyte was suggested as one of the origins of CAFs [67]. Here, GC cells could secrete exosomes, which could induce the transition of pericytes into CAFs, but this experimental result was not proven in GC patients' samples. Bone marrowderived stem cells have also been proposed as the origin of CAFs in GC [68]. This result was proven in the tissues harvested from patients with secondary GC who have previously undergone bone marrow transplantation for various hematologic diseases. Another study insisted that CAFs could be induced from normal resident fibroblasts of the stomach by stimulation of TGF- $\beta$ derived from the scirrhous GC cells [69].

However, in other solid tumors such as those of the head and neck, breast, and pancreas, recent studies show that CAFs contained in one tumor were heterogeneous, presenting different gene expression patterns and a variety of functions $[21,70,71]$. These results imply that CAFs in GC may also include various subtypes that originated from multiple sources, and it would be important to determine which subtype has a crucial role in GC progression. To the best of our knowledge, no study has evaluated the heterogeneity of CAFs in GC; hence, it should be investigated in future studies.

\section{Role of CAF in Gastric Carcinogenesis}

Gastric carcinogenesis is a very complicated process. Because high-throughput genetic profiling in GC tissues did not reveal driver mutation during gastric carcinogenesis [72], the role of environmental factors such as infection and food could be emphasized [73]. Most studies have proposed that those factors could enhance precancerous inflammation in the gastric mucosa, which can lead to GC $[74,75]$.

Although the role of CAF during gastric carcinogenesis has been rarely reported, several candidates derived from 
fibroblasts have been suggested as the contributors to the occurrence of chemically induced GC in murine models $[76,77]$. In one of those models, GC developed in Lewis rats provided with drinking water with $\mathrm{N}$-methyl-N'-nitro$\mathrm{N}$-nitrosoguanidine [77]. During carcinogenesis in this rat model, SPARC-stained fibroblasts appeared in the interstitial portion of early initiation stage of stomach tumors in the test rat; however, this was not observed in the control rat. These results suggested that SPARC-expressing fibroblasts probably contributed to GC development. Hiroto $\mathrm{K}$ et al. studied the role of CAFs on carcinogenesis using N-methyl-Nnitrosourea- (MNU-) induced gastric tumorigenesis mouse model [76]. In this study, compared with normal gastric tissues, IL-6 expression in GC was significantly increased, and IL-6 knockout mouse had a lower incidence of MNUinduced GC than wild-type mice. These results imply that IL6 induced from CAFs has an important role during gastric carcinogenesis.

\section{Role of CAF in GC Invasion and Metastasis}

The invasion and metastasis of cancer cells have long been the causes of death and great challenges for GC patients even after undergoing complex clinical treatments [78]. The poor prognosis and low survival rate of GC patients are mainly due to metastasis [45], and almost $60 \%$ of GC deaths are due to peritoneal recurrence [50]. However, the specific mechanisms of GC metastasis have not been clarified.

The epithelial-mesenchymal transition (EMT) is a biological process by which epithelial cells lose their cell polarity and cell-cell adhesion, gain migratory and invasive capacity, and become resistant to apoptosis. Moreover, the EMT increases the production of components of ECM and gains the invasive properties to become mesenchymal stem cells, which play an important role in the initiation of metastasis during cancer progression [47]. In GC, high-throughput molecular analysis revealed that the expression of EMT gene signature in primary GC was significantly related to poor prognosis [79]. As described earlier, because CAFs were known to advance tumor cell metastasis and invasion by overexpression of a variety of factors that can enhance EMT phenomenon [39], the exploration of communication mechanism between GC cells and CAFs could be crucial in the field of GC metastasis research.

One study described that the effect of CAFs on increased migration of GC cells was more significant than normal tissue-associated fibroblasts. This study suggested that microRNA-106b is a CAF-specific maker and has a crucial role in the reinforcement of phosphatase and tensin (PTEN) signaling in GC cells [40]. While this study did not indicate the exact communicators between GC cells and CAFs, Wu X et al. [41] showed that GC-derived CAFs secrete significant quantities of IL-6, which can induce EMT phenomenon and increase migration of GC cells through activation of Janus kinase 2/signal transducers and activators of transcription (JAK2/STAT3) pathway in GC cells. In addition, they showed that deprivation of IL- 6 by inhibiting the JAK/STAT3 pathway with a specific inhibitor markedly diminishes these phenotypes in GC cells induced by CAFs. Another secreted factor such as fibroblast growth factor 9 (FGF-9) could be suggested as a communicator between GC cells and CAFs [42]. This study showed that the CAFs isolated from GC tissues could secrete FGF-9 into the extracellular area under the regulation of miR-214 and the secreted FGF-9 could induce EMT in GC cells. CAFderived exosomes could be a key player in the communication between GC cells cultured from scirrhous type GC, which is a subtype of GC with abundant fibrotic stroma [37] because exosomes are cell-derived vesicles containing functional biomolecules that can be transferred to recipient cells [43]. In particular, CD9 is a specific marker of exosomes that originated from CAFs, and CD9 exosomes from CAFs could increase the migration and invasion ability of GC cells.

Taken together, the evidence suggests that CAFs may play a pivotal role in the migration and invasion of GC cells. In addition, other factors such as stromal derived factor 1 (SDR1), CXCL12, and interleukin 11 have been suggested as CAF-derived inducers for migration and invasion of GC $[36,38,44,46,48,49,80,81]$, and their mechanisms are listed in Table 1. The mechanisms associated with CAF-induced motility of GC cells could be a novel target in the treatment of GC.

\section{Role of CAF in Angiogenesis of GC}

Pathological angiogenesis is a hallmark of cancer [82]. Growth, invasion, and metastasis of malignant tumors depend on neovascularization that is controlled by proangiogenic and antiangiogenic elements [83, 84]. Past studies have shown the positive correlation between the expression of factors related to tumor angiogenesis and poor clinical outcomes of GC patients $[85,86]$. Moreover, antiangiogenic agent, a monoclonal antibody VEGFR2 antagonist, is one of the few targeted agents showing clinical benefit in metastatic GC patients [87]. A plethora of factors have been proposed as contributors to angiogenesis, but major factors should be clarified before administering novel targeted agents to block GC angiogenesis.

Increasing evidence has shown that chemokine secretion by CAFs may support the recruitment of bone marrowderived angiogenic cells [58]. CAFs may be a major source of angiogenic factors [88]. In GC, galectin-1 [89] and hepatocyte growth factor (HGF) [90] have been proposed as CAFderived secretory proteins, which contribute to GC angiogenesis. Galectin expression in CAFs was positively related to increased expression of endothelial cell marker, CD31 [89]. Ding X et al. [90] discovered that the phosphorylation of Akt and ERK1/2 was increased in GC cells treated with HGF and cocultured with CAFs. Both Akt inhibitors and ERK1/2 inhibitors reduced the angiogenic and vasculogenic abilities of HGF. However, these results have been confirmed using an in vitro angiogenesis assay (tube formation assay). To elucidate the correlation between CAFs and GC angiogenesis, the inhibitory effects of CAF-derived proteins on GC 


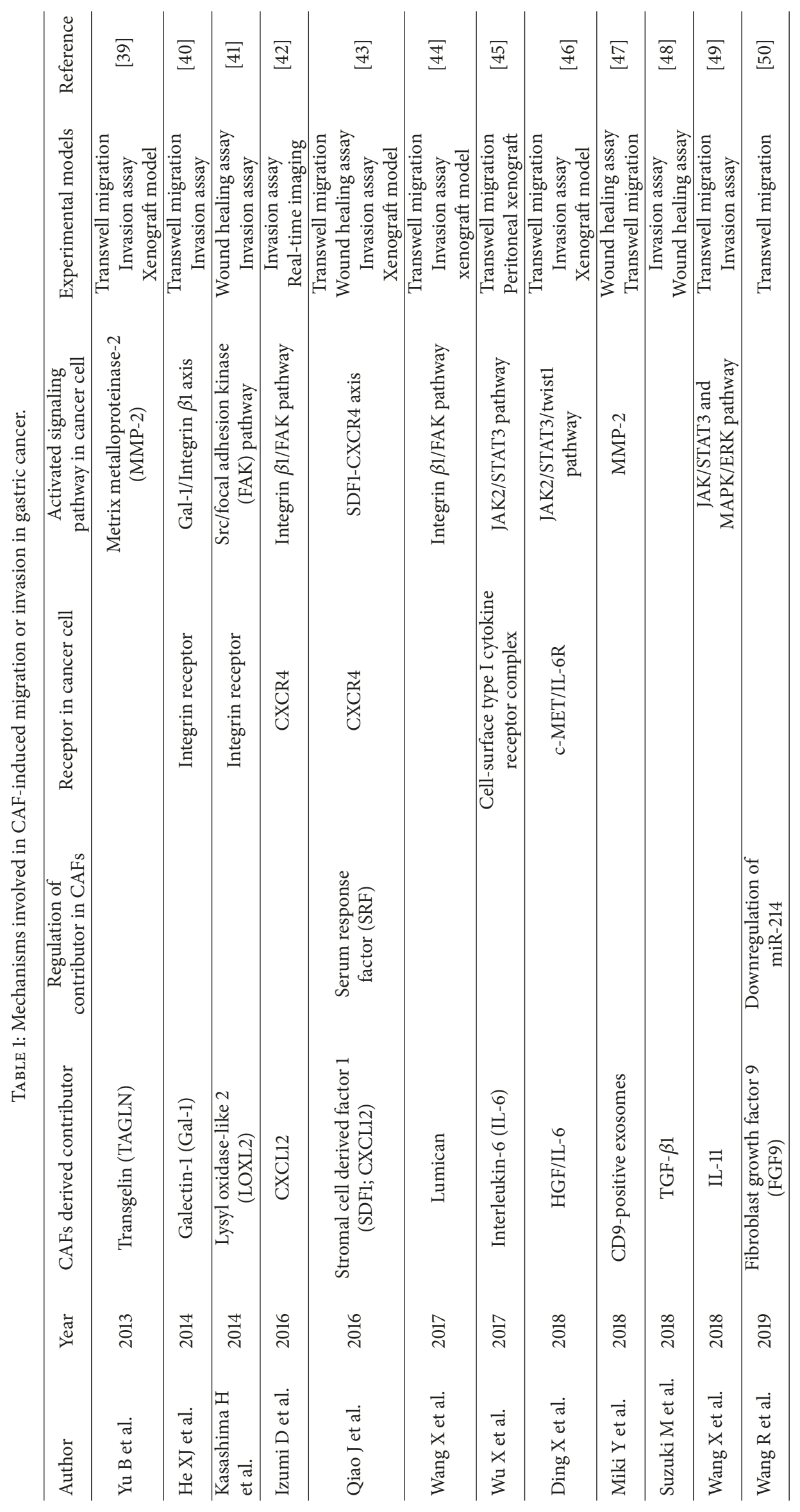


angiogenesis should be investigated in GC animal models for clinical application.

\section{Role of CAF in GC Resistance to Chemotherapy}

Chemotherapies for GC have shown some clinical effects; however, some patients still show progression and recurrence after chemotherapy in clinical settings, and there are many obstacles to overcome this issue. One of the commonly reported reasons for failed chemotherapy in clinics is the occurrence of drug resistance. Unfortunately, the underlying mechanism of multidrug resistance in GC remains unclear.

Previous studies evaluating chemotherapy resistance have focused on the tumor microenvironment. In particular, cancer cell-ECM interactions, CAF-ECM adhesion, and cytokine or chemokine-mediated signaling pathways have been considered as TME-related resistance to chemotherapy [91, 92]; CAFs may have a major role in those mechanisms. CAFs have been confirmed to regulate chemoresistance by secreting cytokines, including stromal cell-derived factor- $1 \alpha$, IL-6, and IL-7 [93-95], and may also increase intratumoral interstitial fluid pressure, thus indirectly inhibiting the uptake of anticancer drugs [96]. However, the role of CAF in chemoresistance has not been clarified.

Only one study was published describing that CAFs secreting IL-11 could contribute to resistance to combined chemotherapy regimens in GC cells by activating gp130/JAK/STAT3/Bcl signaling pathway [97]. Recently, our group investigated the GC CAF-specific secretory protein involved in chemoresistance [98]. Through the analysis of transcriptome between fibroblasts from paired normal gastric and GC tissues, IL-6 was suggested as a CAF-specific cytokine. In addition, transcriptome data and immunohistochemical staining for GC tissues revealed that IL-6 was usually expressed in the fibrotic stromal cell. CAF-derived IL-6 could induce resistance to 5 -FU or cisplatin in various experimental models, such as in vitro and in vivo xenograft, and tocilizumab, a monoclonal antibody that inhibits the binding of IL-6 to its receptor, effectively suppressed the development of drug resistance. If those results were applied in the clinical setting, it could have prevented the occurrence of chemoresistance in GC patients.

\section{Role of CAF in Tumor Immunity of GC}

The Cancer Genome Atlas (TCGA) project for GC revealed four molecular subtypes [72]; among them, Epstein-Barr virus- (EBV-) positive and microsatellite instability subtype was associated with high-density tumor-infiltrating lymphocytes and showed a better prognosis compared with other subtypes [99]. Some previous studies have reported that infiltrating immune cells had an effective host immune response against GC cells [100, 101]. Taken together, the tumor escape from immune response could deteriorate the outcome of GC patients; therefore, this mechanism could be a good target to improve the patients' prognosis. However, the exact mechanisms involved remain unknown.

CAFs produce a plethora of cytokines and chemokines potentially contributing to tumor immunity at various stages of cancer progression. The direct or indirect effects of IL-6, IL-8, IL-10, TGF- $\beta$, C-C motif chemokine ligand 2 (CCL2), C-X-C motif chemokine ligand 9 (CXCL9), and CXCL10, but not limited to those, on tumor immunity in patients with oral, breast, and pancreatic cancer have been investigated [102104]. The role of CAFs in the regulation of tumor immunity is seldom reported in GC. However, recent studies show that CAFs were deeply involved in M2 polarization of macrophage suppressing immune clearance $[105,106]$. CAFs could induce M2 polarization in tumor-associated macrophage (TAM); it has been well reported that the accumulation of M2 macrophage was significantly related to the poor survival of GC patients [107] and M2 macrophages directly induced invasion and metastasis of GC cells or indirectly reduced immune response within GC tumors. The proportion of CAF in the deep portion of the primary GC is higher than that in the superficial layer, which positively correlates with the increased number of M2 macrophages [105]. Other studies reported that neurooncological ventral antigen 1 (NOVA1), a marker of activated CAFs, was suppressed in GC microenvironment including CAFs, and NOVA1 suppression was significantly correlated with immune dysfunction such as an accumulation of M2 macrophage [106]. Although several secretory proteins such as macrophage colony-stimulating factor (M-CSF) [108], interleukin 33 (IL-33) [109], CCL2, and interleukin 6 (IL-6) [110] have been suggested as stimulators derived from CAFs for M2 macrophage in esophageal and pancreatic cancers, it has never been reported in GC and should be discovered to be applied for clinical setting in the future.

\section{CAF-Targeting Agents}

As the role of CAFs in the progression of solid tumors becomes clearer, several therapeutic approaches to inhibit the function of CAFs have been suggested as novel agents.

Some of those CAF-targeting agents have been already applied in clinical settings in patients with various malignant or nonmalignant diseases, but they were not used as CAF inhibitors. Nilotinib is an inhibitor of the c-KIT receptor and is effective in the treatment of chronic myeloid leukemia, melanoma, and gastrointestinal stromal tumors [111-113]. Aside from c-KIT receptors, nilotinib also inhibits other receptor tyrosine kinases such as platelet-derived growth factor receptors (PDGF-R $\alpha$ and PDGF-R $\beta$ ) or discoidin domain receptors (DDR1 or DDR2) $[52,114]$. A previous study reported that PDGF-R was expressed in CAFs, not in cancer cells [60]. As activated PDGF-R signaling pathway in tumor stroma can increase the proliferation of cancer cells [57] and stimulate GC angiogenesis [61], nilotinib could be used as a potential inhibitor for GCs with a profuse fibrotic stroma [51]. Tocilizumab has been clinically used in several patients with rheumatic disease as an inhibitor 


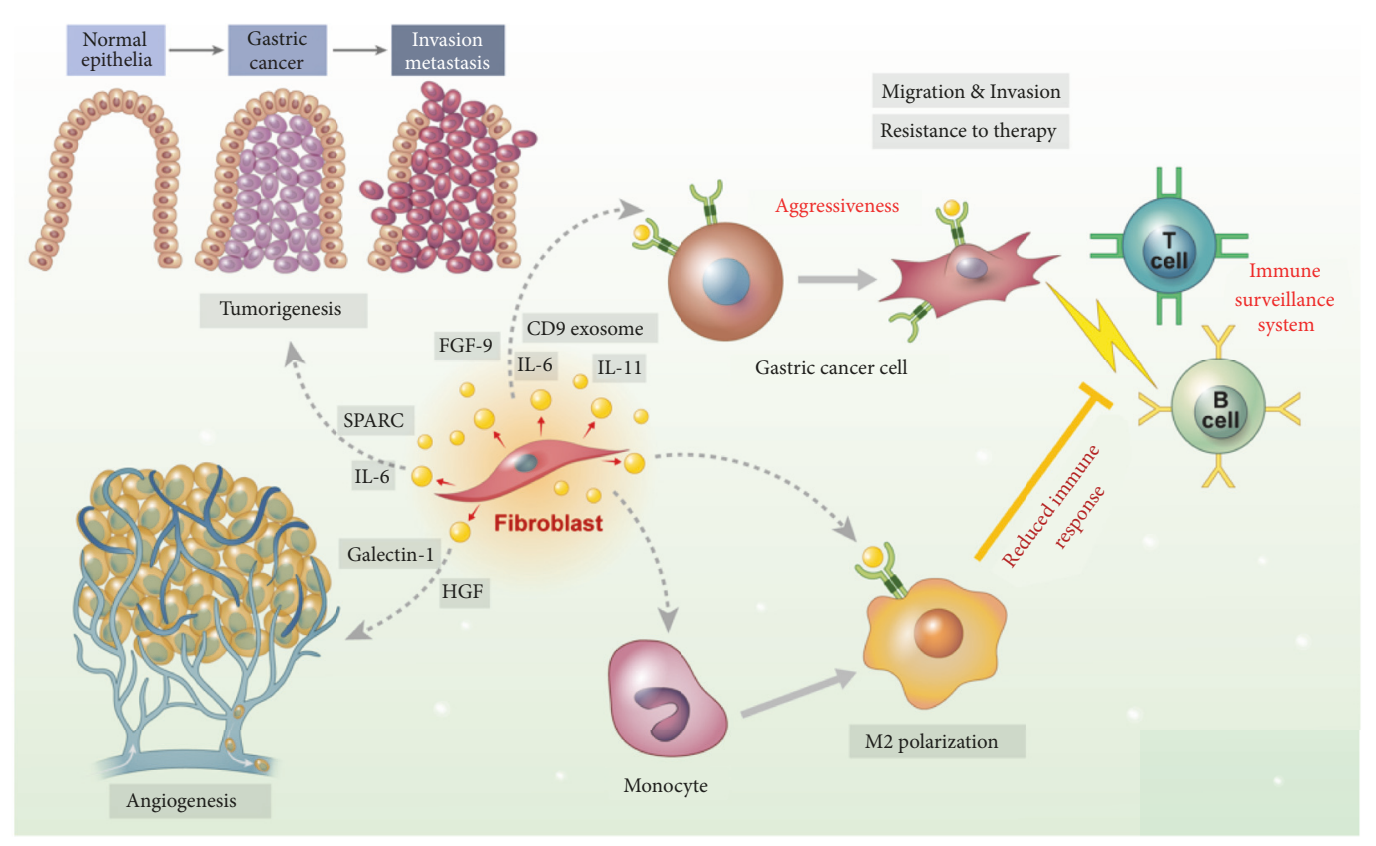

FIGURE 1: Schematic image for role of cancer-associated fibroblasts in tumor microenvironment of gastric cancer.

of a pleiotropic cytokine such as IL-6. This drug has been proposed as a potential inhibitor of GC cells and CAFs. The in silico analysis using TCGA database of GCs revealed that low expression of both $I L-6$ and $I L-6 R$ genes was significantly related to improved survival of GC patients and the in vivo experiments described that tocilizumab could efficiently reduce tumor growth in xenograft models of GC cells mixed with CAFs [53].

Several natural products have been proposed as suppressors of CAF activity. Astragaloside IV, the main component of nontoxic Chinese herb, inhibited cellular migration by reducing the ability of CAFs to promote GC cell migration and invasion through regulation of microRNA such as miR214 in the nontoxic low dose [54]. Paeoniflorin, the principal bioactive component of Radix Paeoniae Rubra, inhibited the secretion of IL- 6 from CAFs and consequently inhibited the migration- and invasion-promoting capacities of GC CAFs [55].

Other agents have been evaluated to determine their efficacy in suppressing the migration- and invasionpromoting capacities of GC CAFs through various preclinical models $[46,56,59,62,115-118]$; these agents are listed in Table 2. However, future studies are required to determine the toxic effects and indications of those agents.

\section{Conclusion and Future Perspectives}

Studies have shown that the CAFs are an important component in the TME of GC, and previous studies revealed the potential effects of CAFs including carcinogenesis, metastasis, invasion, angiogenesis, resistance to therapy, and tumor immunity in various GC models (Figure 1). However, the inhibitory mechanism of CAFs on GC cells as well as TME has not been applied in GC treatment. Moreover, the specific markers and origin of CAFs remain controversial. Recent advanced technologies for single-cell transcriptome profiling have uncovered spatial, functional, and genomic heterogeneity of cancer cells and associated host cells in TME [119]. The single-cell RNA-sequencing for lung [120], pancreas [121], and colorectal cancer [122] revealed that CAFs in solid tumors have molecular and functional intra- and interheterogeneity and suggested specific CAF subpopulations as targets for cancer treatment. However, to the best of our knowledge, there has been no report that studies CAFs heterogeneity through the single-cell molecular profiling in GCs. Considering the functional role of CAFs in GCs, further studies evaluating CAF heterogeneity are warranted to determine the critical CAF subtype that expresses specific targets for GC treatment.

\section{Conflicts of Interest}

The authors declare that they have no conflicts of interest.

\section{Acknowledgments}

This study was supported by the Basic Science Research Program through the National Research Foundation of Korea, funded by the Ministry of Education (2016R1D1A1B03933083 


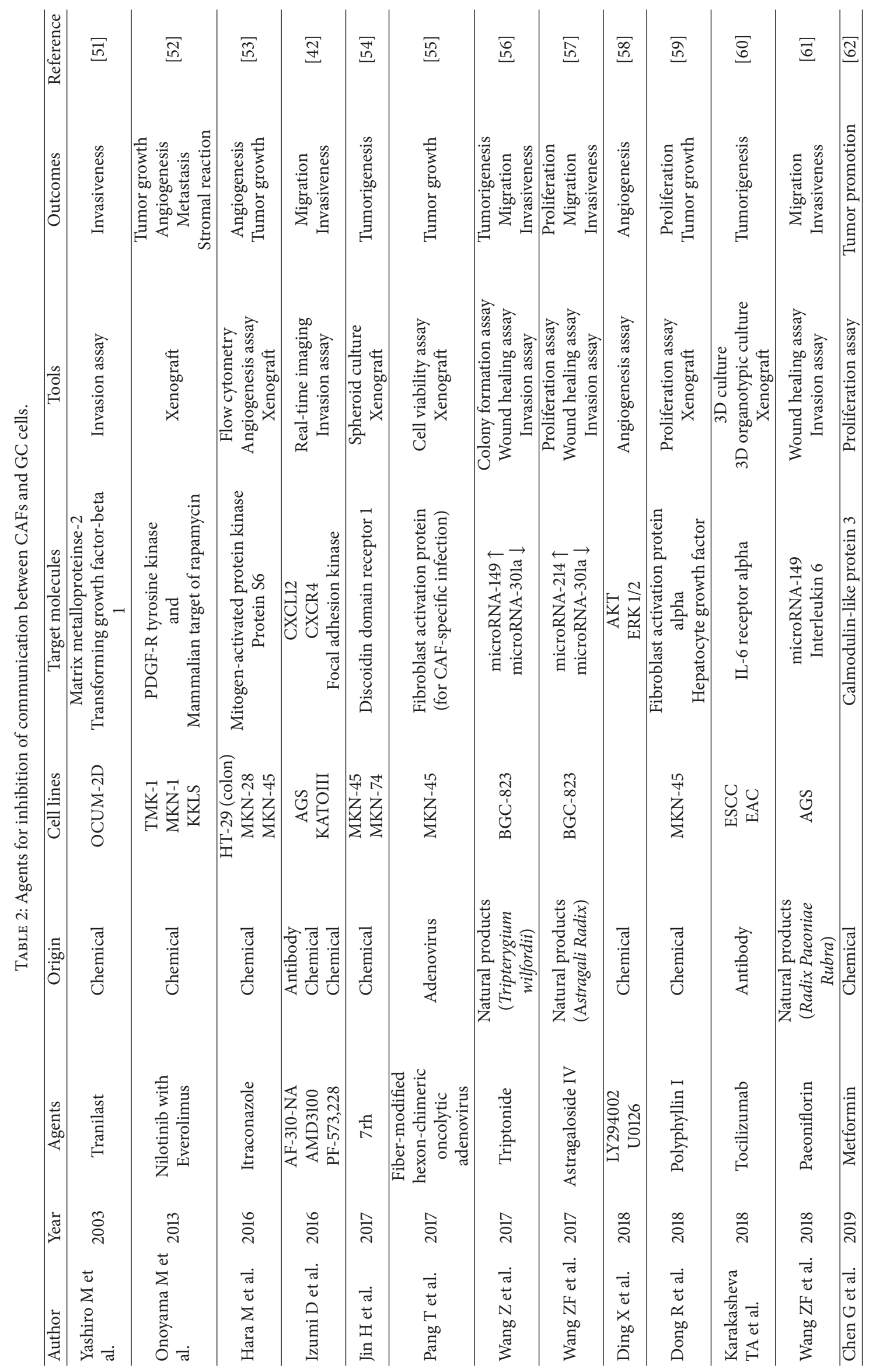


and 2018R1A6A3A01012164), funded by a grant from the Korea Health Technology R\&D Project through the Korea Health Industry Development Institute, and funded by the Ministry of Health \& Welfare, Republic of Korea (HI17C0364).

\section{References}

[1] P. Cirri and P. Chiarugi, "Cancer-associated-fibroblasts and tumour cells: A diabolic liaison driving cancer progression," Cancer and Metastasis Reviews, vol. 31, no. 1-2, pp. 195-208, 2012.

[2] J. A. Ajani, T. A. D’Amico, K. Almhanna et al., "Gastric cancer, version 3.2016, NCCN clinical practice guidelines in oncology," Journal of the National Comprehensive Cancer Network, vol. 14, no. 10, pp. 1286-1312, 2016.

[3] Japanese Gastric Cancer Association, "Japanese gastric cancer treatment guidelines 2014 (ver. 4)," Gastric Cancer, vol. 20, no. 1, pp. 1-19, 2017.

[4] H. In, I. Solsky, B. Palis, M. Langdon-Embry, J. Ajani, and T. Sano, "Validation of the 8th edition of the AJCC TNM staging system for gastric cancer using the national cancer database," Annals of Surgical Oncology, vol. 24, no. 12, pp. 3683-3691, 2017.

[5] S. G. Kim, H. S. Seo, H. H. Lee, K. Y. Song, and C. H. Park, "Comparison of the differences in survival rates between the 7th and 8th editions of the ajcc tnm staging system for gastric adenocarcinoma: A single-institution study of 5,507 patients in korea," Gastric Cancer, vol. 17, no. 3, pp. 212-219, 2017.

[6] T. Sano, D. G. Coit, H. H. Kim et al., "Proposal of a new stage grouping of gastric cancer for TNM classification: International Gastric Cancer Association staging project," Gastric Cancer, vol. 20, no. 2, pp. 217-225, 2017.

[7] J. A. Ajani, W. Rodriguez, G. Bodoky et al., "Multicenter phase III comparison of cisplatin/S-1 with cisplatin/infusional fluorouracil in advanced gastric or gastroesophageal adenocarcinoma study: The FLAGS trial," Journal of Clinical Oncology, vol. 28, no. 9, pp. 1547-1553, 2010.

[8] N. Boku, S. Yamamoto, H. Fukuda et al., "Fluorouracil versus combination of irinotecan plus cisplatin versus S-1 in metastatic gastric cancer: a randomised phase 3 study," The Lancet Oncology, vol. 10, no. 11, pp. 1063-1069, 2009.

[9] Y.-K. Kang, W.-K. Kang, D.-B. Shin et al., "Capecitabine/ cisplatin versus 5-fluorouracil/cisplatin as first-line therapy in patients with advanced gastric cancer: a randomised phase III noninferiority trial," Annals of Oncology, vol. 20, no. 4, pp. 666673, 2009.

[10] Y. Yamada, K. Higuchi, K. Nishikawa et al., "Phase III study comparing oxaliplatin plus S-1 with cisplatin plus S-1 in chemotherapy-naive patients with advanced gastric cancer," Annals of Oncology, vol. 26, no. 1, pp. 141-148, 2014.

[11] W. Xu, Z. Yang, and N. Lu, "Molecular targeted therapy for the treatment of gastric cancer," Journal of Experimental \& Clinical Cancer Research, vol. 35, p. 1, 2016.

[12] T. Ishimoto, H. Sawayama, H. Sugihara, and H. Baba, "Interaction between gastric cancer stem cells and the tumor microenvironment," Journal of Gastroenterology, vol. 49, no. 7, pp. 11111120, 2014.

[13] H.-Y. Ma, X.-Z. Liu, and C.-M. Liang, "Inflammatory microenvironment contributes to epithelial-mesenchymal transition in gastric cancer," World Journal of Gastroenterology, vol. 22, no. 29, pp. 6619-6628, 2016.
[14] M. Yashiro and K. Hirakawa, "Cancer-stromal interactions in scirrhous gastric carcinoma," Cancer Microenvironment, vol. 3, no. 1, pp. 127-135, 2010.

[15] R. Kalluri, "The biology and function of fibroblasts in cancer," Nature Reviews Cancer, vol. 16, no. 9, pp. 582-598, 2016.

[16] Y. Kojima, A. Acar, E. N. Eaton et al., "Autocrine TGF- $\beta$ and stromal cell-derived factor-1 (SDF-1) signaling drives the evolution of tumor-promoting mammary stromal myofibroblasts," Proceedings of the National Acadamy of Sciences of the United States of America, vol. 107, no. 46, pp. 20009-20014, 2010.

[17] D. Fukumura, R. Xavier, T. Sugiura et al., "Tumor induction of VEGF promoter activity in stromal cells," Cell, vol. 94, no. 6, pp. 715-725, 1998.

[18] J. Tommelein, L. Verset, T. Boterberg, P. Demetter, M. Bracke, and O. De Wever, "Cancer-associated fibroblasts connect metastasis-promoting communication in colorectal cancer," Frontiers in Oncology, vol. 5, p. 63, 2015.

[19] A. Cruz-Bermúdez, R. Laza-Briviesca, R. J. Vicente-Blanco et al., "Cancer-associated fibroblasts modify lung cancer metabolism involving ROS and TGF- $\beta$ signaling," Free Radical Biology \& Medicine, vol. 130, pp. 163-173, 2019.

[20] E. Y. T. Lau, J. Lo, B. Y. L. Cheng et al., “Cancer-associated fibroblasts regulate tumor-initiating cell plasticity in hepatocellular carcinoma through c-Met/FRA1/HEY1 signaling," Cell Reports, vol. 15, no. 6, pp. 1175-1189, 2016.

[21] S. Su, J. Chen, H. Yao et al., "CD10(+)GPR77(+) cancerassociated fibroblasts promote cancer formation and chemoresistance by sustaining cancer stemness," Cell, vol. 172, no. 4, pp. 841-856, 2018.

[22] J. Paulsson, L. Ryden, C. Strell et al., "High expression of stromal PDGFRbeta is associated with reduced benefit of tamoxifen in breast cancer," Journal of Pathology: Clinical Research, vol. 3, no. 1, pp. 38-43, 2017.

[23] A. Orimo, P. B. Gupta, D. C. Sgroi et al., "Stromal fibroblasts present in invasive human breast carcinomas promote tumor growth and angiogenesis through elevated SDF-1/CXCL12 secretion," Cell, vol. 121, no. 3, pp. 335-348, 2005.

[24] F. Calvo, N. Ege, A. Grande-Garcia et al., "Mechanotransduction and YAP-dependent matrix remodelling is required for the generation and maintenance of cancer-associated fibroblasts," Nature Cell Biology, vol. 15, no. 6, pp. 637-646, 2013.

[25] M.-G. Procopio, C. Laszlo, D. Al Labban et al., "Combined CSL and p53 downregulation promotes cancer-associated fibroblast activation," Nature Cell Biology, vol. 17, no. 9, pp. 1193-1204, 2015.

[26] M. T. Dimanche-Boitrel, L. Vakaet, P. Pujuguet et al., "In vivo and in vitro invasiveness of a rat colon-cancer cell line maintaining E-cadherin expression: An enhancing role of tumor-associated myofibroblasts," International Journal of Cancer, vol. 56, no. 4, pp. 512-521, 1994.

[27] E. M. de Kruijf, J. G. H. van Nes, C. J. H. van de Velde et al., "Tumor-stroma ratio in the primary tumor is a prognostic factor in early breast cancer patients, especially in triple-negative carcinoma patients," Breast Cancer Research and Treatment, vol. 125, no. 3, pp. 687-696, 2011.

[28] Z. Lv, X. Cai, X. Weng et al., "Tumor-stroma ratio is a prognostic factor for survival in hepatocellular carcinoma patients after liver resection or transplantation," Surgery, vol. 158, no. 1, pp. 142-150, 2015.

[29] W. E. Mesker, J. M. C. Junggeburt, K. Szuhai et al., "The carcinoma-stromal ratio of colon carcinoma is an independent factor for survival compared to lymph node status and tumor stage," Cellular Oncology, vol. 29, no. 5, pp. 387-398, 2007. 
[30] K. Wang, W. Ma, J. Wang et al., "Tumor-stroma ratio is an independent predictor for survival in esophageal squamous cell carcinoma," Journal of Thoracic Oncology, vol. 7, no. 9, pp. 14571461, 2012.

[31] E. Y. Kim, H. M. Yoo, K. Y. Song, and C. H. Park, "Limited significance of curative surgery in Borrmann type IV gastric cancer," Medical Oncology, vol. 33, no. 7, p. 69, 2016.

[32] K. Kitamura, R. Beppu, H. Anai et al., "Clinicopathologic study of patients with Borrmann type iv gastric carcinoma," Journal of Surgical Oncology, vol. 58, no. 2, pp. 112-117, 1995.

[33] J. H. Yook, S. T. Oh, and B. S. Kim, “Clinicopathological analysis of borrmann Type IV gastric cancer," Cancer Research and Treatment, vol. 37, no. 2, pp. 87-91, 2005.

[34] Y. Wu, H. Grabsch, T. Ivanova et al., "Comprehensive genomic meta-analysis identifies intra-tumoural stroma as a predictor of survival in patients with gastric cancer," Gut, vol. 62, no. 8, pp. 1100-1111, 2013.

[35] D. Lee, I.-H. Ham, S. Y. Son, S.-U. Han, Y.-B. Kim, and H. Hur, "Intratumor stromal proportion predicts aggressive phenotype of gastric signet ring cell carcinomas," Gastric Cancer, vol. 20, no. 4, pp. 591-601, 2017.

[36] X. He, H. Tao, Z. Hu et al., "Expression of galectin-1 in carcinoma-associated fibroblasts promotes gastric cancer cell invasion through upregulation of integrin $\beta 1$," Cancer Science, vol. 105, no. 11, pp. 1402-1410, 2014.

[37] Y. Miki, M. Yashiro, T. Okuno et al., "CD9-positive exosomes from cancerassociated fibroblasts stimulate the migration ability of scirrhous-type gastric cancer cells," British Journal of Cancer, vol. 118, no. 6, pp. 867-877, 2018.

[38] B. Yu, X. Chen, J. Li et al., "Stromal fibroblasts in the microenvironment of gastric carcinomas promote tumor metastasis via upregulating TAGLN expression," BMC Cell Biology, vol. 14, no. 1, p. 17, 2013.

[39] Y.-Q. Lan, L.-J. Kong, X.-Y. Lin et al., "Combination chemotherapy with paclitaxel and oxaliplatin as first-line treatment in patients with advanced gastric cancer," Cancer Chemotherapy and Pharmacology, vol. 81, no. 6, pp. 1007-1015, 2018.

[40] T.-S. Yang, X.-H. Yang, X. Chen et al., "MicroRNA-106b in cancer-associated fibroblasts from gastric cancer promotes cell migration and invasion by targeting PTEN," FEBS Letters, vol. 588, no. 13, pp. 2162-2169, 2014.

[41] X. Wu, P. Tao, Q. Zhou et al., "IL-6 secreted by cancer-associated fibroblasts promotes epithelial-mesenchymal transition and metastasis of gastric cancer via JAK2/STAT3 signaling pathway," Oncotarget, vol. 8, no. 13, pp. 20741-20750, 2017.

[42] R. Wang, Y. Sun, W. Yu et al., "Downregulation of miRNA214 in cancer-associated fibroblasts contributes to migration and invasion of gastric cancer cells through targeting FGF9 and inducing EMT," Journal of Experimental \& Clinical Cancer Research, vol. 38, no. 1, p. 20, 2019.

[43] H. Valadi, K. Ekström, A. Bossios, M. Sjöstrand, J. J. Lee, and J. O. Lötvall, "Exosome-mediated transfer of mRNAs and microRNAs is a novel mechanism of genetic exchange between cells," Nature Cell Biology, vol. 9, no. 6, pp. 654-659, 2007.

[44] H. Kasashima, M. Yashiro, H. Kinoshita et al., "Lysyl oxidaselike 2 (LOXL2) from stromal fibroblasts stimulates the progression of gastric cancer," Cancer Letters, vol. 354, no. 2, pp. 438446, 2014.

[45] N. Oue, P. A. Phyu, Y. Mitani, H. Kuniyasu, H. Nakayama, and W. Yasui, "Genes involved in invasion and metastasis of gastric cancer identified by array-based hybridization and serial analysis of gene expression," Oncology, vol. 69, no. 1, pp. 17-22, 2005.

[46] D. Izumi, T. Ishimoto, K. Miyake et al., "CXCL12/CXCR4 activation by cancer-associated fibroblasts promotes integrin $\beta 1$ clustering and invasiveness in gastric cancer," International Journal of Cancer, vol. 138, no. 5, pp. 1207-1219, 2016.

[47] G. Krzysiek-Maczka, A. Targosz, U. Szczyrk et al., "Role of helicobacter pylori infection in cancer-associated fibroblastinduced epithelial-mesenchymal transition in vitro," Helicobacter, vol. 23, no. 6, Article ID e12538, 2018.

[48] J. Qiao, Z. Liu, C. Yang, L. Gu, and D. Deng, "SRF promotes gastric cancer metastasis through stromal fibroblasts in an SDF1-CXCR4-dependent manner," Oncotarget, vol. 7, no. 29, pp. 46088-46099, 2016.

[49] L. Su, X. Wang, Q. Zhou, J. Li, and B. Liu, "Cancer-associated fibroblast-derived lumican promotes gastric cancer progression via the integrin betal-FAK signalling pathway," International Journal of Cancer, vol. 141, no. 5, pp. 998-1010, 2017.

[50] Y. Yonemura, Y. Endou, M. Shinbo et al., "Safety and efficacy of bidirectional chemotherapy for treatment of patients with peritoneal dissemination from gastric cancer: Selection for cytoreductive surgery," Journal of Surgical Oncology, vol. 100, no. 4, pp. 311-316, 2009.

[51] M. Onoyama, Y. Kitadai, Y. Tanaka et al., "Combining molecular targeted drugs to inhibit both cancer cells and activated stromal cells in gastric cancer," Neoplasia (United States), vol. 15, no. 12, pp. 1391-1399, 2013.

[52] J. A. Fletcher, "Role of KIT and platelet-derived growth factor receptors as oncoproteins," Seminars in Oncology, vol. 31, Supplement 6, no. 2, pp. 4-11, 2004.

[53] T. A. Karakasheva, E. W. Lin, Q. Tang et al., "IL-6 mediates cross-talk between tumor cells and activated fibroblasts in the tumor microenvironment," Cancer Research, vol. 78, no. 17, pp. 4957-4970, 2018.

[54] Z. F. Wang, D. G. Ma, and Z. Zhu, "Astragaloside IV inhibits pathological functions of gastric cancer-associated fibroblasts," World Journal of Gastroenterology, vol. 23, no. 48, pp. 8512-8525, 2017.

[55] Z. Wang, D. Ma, L. Wang et al., "Paeoniflorin inhibits migration- and invasion-promoting capacities of gastric cancer associated fibroblasts," Chinese Journal of Integrative Medicine, 2018.

[56] M. Yashiro, K. Murahashi, T. Matsuoka et al., "Tranilast (N3,4 -dimethoxycinamoyl anthranilic acid): a novel inhibitor of invasion-stimulating interaction between gastric cancer cells and orthotopic fibroblasts," Anticancer Reseach, vol. 23, no. 5A, pp. 3899-3904, 2003.

[57] A. Östman, "PDGF receptors-mediators of autocrine tumor growth and regulators of tumor vasculature and stroma," Cytokine \& Growth Factor Reviews, vol. 15, no. 4, pp. 275-286, 2004.

[58] E. M. De Francesco, R. Lappano, M. F. Santolla, S. Marsico, A. Caruso, and M. Maggiolini, "HIF- $1 \alpha /$ GPER signaling mediates the expression of VEGF induced by hypoxia in breast cancer associated fibroblasts (CAFs)," Breast Cancer Research, vol. 15, no. 4, p. R64, 2013.

[59] M. Hara, T. Nagasaki, K. Shiga, and H. Takeyama, "Suppression of cancer-associated fibroblasts and endothelial cells by itraconazole in bevacizumab-resistant gastrointestinal cancer," Anticancer Reseach, vol. 36, no. 1, pp. 169-177, 2016.

[60] T. Sumida, Y. Kitadai, K. Shinagawa et al., "Anti-stromal therapy with imatinib inhibits growth and metastasis of gastric 
carcinoma in an orthotopic nude mouse model," International Journal of Cancer, vol. 128, no. 9, pp. 2050-2062, 2011.

[61] W. Risau, H. Drexler, V. Mironov et al., "Platelet-derived growth factor is angiogenic in vivo," Growth Factors, vol. 7, no. 4, pp. 261-266, 1992.

[62] H. Jin, I.-H. Ham, H. J. Oh et al., "Inhibition of discoidin domain receptor 1 prevents stroma-induced peritoneal metastasis in gastric carcinoma," Molecular Cancer Research, vol. 16, no. 10, pp. 1590-1600, 2018.

[63] N. A. Bhowmick, E. G. Neilson, and H. L. Moses, "Stromal fibroblasts in cancer initiation and progression," Nature, vol. 432, no. 7015, pp. 332-337, 2004.

[64] K. Hosaka, Y. Yang, T. Seki et al., "Pericyte-fibroblast transition promotes tumor growth and metastasis," Proceedings of the National Acadamy of Sciences of the United States of America, vol. 113, no. 38, pp. E5618-E5627, 2016.

[65] M. Wawro, K. Chojnacka, K. Wieczorek-Szukała, K. Sobierajska, and J. Niewiarowska, "Invasive colon cancer cells induce transdifferentiation of endothelium to cancer-associated fibroblasts through microtubules enriched in tubulin- $\beta 3$," International Journal of Molecular Sciences, vol. 20, no. 1, p. 53, 2019.

[66] L. T. McDonald, D. L. Russell, R. R. Kelly et al., "Hematopoietic stem cell-derived cancer-associated fibroblasts are novel contributors to the pro-tumorigenic microenvironment," Neoplasia, vol. 17, no. 5, pp. 434-448, 2015.

[67] X. Ning, H. Zhang, C. Wang, and X. Song, "Exosomes released by gastric cancer cells induce transition of pericytes into cancerassociated fibroblasts," Medical Science Monitor, vol. 24, pp. 2350-2359, 2018.

[68] D. L. Worthley, A. Ruszkiewicz, R. Davies et al., "Human gastrointestinal neoplasia-associated myofibroblasts can develop from bone marrow-derived cells following allogeneic stem cell transplantation," Stem Cells, vol. 27, no. 6, pp. 1463-1468, 2009.

[69] Y. Fuyuhiro, M. Yashiro, S. Noda et al., "Upregulation of cancerassociated myofibroblasts by TGF- $\beta$ from scirrhous gastric carcinoma cells," British Journal of Cancer, vol. 105, no. 7, pp. 996-1001, 2011.

[70] A. Costa, Y. Kieffer, A. Scholer-Dahirel et al., "Fibroblast heterogeneity and immunosuppressive environment in human breast cancer," Cancer Cell, vol. 33, no. 3, pp. 463-479, 2018.

[71] A. Givel, Y. Kieffer, A. Scholer-Dahirel et al., "miR200-regulated CXCL12 $\beta$ promotes fibroblast heterogeneity and immunosuppression in ovarian cancers," Nature Communications, vol. 9, no. 1, p. 1056, 2018.

[72] The Cancer Genome Atlas Research Network, "Comprehensive molecular characterization of gastric adenocarcinoma," Nature, vol. 513, no. 7517, pp. 202-209, 2014.

[73] C. de Martel, D. Forman, and M. Plummer, "Gastric cancer: epidemiology and risk factors," Gastroenterology Clinics of North America, vol. 42, no. 2, pp. 219-240, 2013.

[74] F. Wang, W. Meng, B. Wang, and L. Qiao, "Helicobacter pylori-induced gastric inflammation and gastric cancer," Cancer Letters, vol. 345, no. 2, pp. 196-202, 2014.

[75] K. Senol, M. B. Ozkan, S. Vural et al., "The role of inflammation in gastric cancer," Inflammation and Cancer, vol. 816, pp. 235257, 2014.

[76] H. Kinoshita, Y. Hirata, H. Nakagawa et al., "Interleukin-6 mediates epithelial-stromal interactions and promotes gastric tumorigenesis," PLoS ONE, vol. 8, no. 4, Article ID e60914, 2013.
[77] H. Soda, M. Oka, M. Soda et al., "Appearance of osteonectinexpressing fibroblastic cells in early rat stomach carcinogenesis and stomach tumors induced with $\mathrm{N}$-methyl-N'-nitro-Nnitrosoguanidine," Japanese Journal of Cancer Research, vol. 93, no. 9, pp. 960-967, 2002.

[78] J. Liu, X. Geng, and Y. Li, "Milky spots: omental functional units and hotbeds for peritoneal cancer metastasis," Tumor Biology, vol. 37, no. 5, pp. 5715-5726, 2016.

[79] R. Cristescu, J. Lee, M. Nebozhyn et al., "Molecular analysis of gastric cancer identifies subtypes associated with distinct clinical outcomes," Nature Medicine, vol. 21, no. 5, pp. 449-456, 2015.

[80] X. Ding, J. Ji, J. Jiang et al., "HGF-mediated crosstalk between cancer-associated fibroblasts and MET-unamplified gastric cancer cells activates coordinated tumorigenesis and metastasis," Cell Death \& Disease, vol. 9, no. 9, p. 867, 2018.

[81] M. Suzuki, T. Yokobori, N. Gombodorj et al., "High stromal transforming growth factor $\beta$-induced expression is a novel marker of progression and poor prognosis in gastric cancer," Journal of Surgical Oncology, vol. 118, no. 6, pp. 966-974, 2018.

[82] P. Carmeliet and R. K. Jain, "Angiogenesis in cancer and other diseases," Nature, vol. 407, no. 6801, pp. 249-257, 2000.

[83] R. T.-P. Poon, S.-T. Fan, and J. Wong, "Clinical implications of circulating angiogenic factors in cancer patients," Journal of Clinical Oncology, vol. 19, no. 4, pp. 1207-1225, 2001.

[84] J. Folkman, "What is the evidence that tumors are angiogenesis dependent?" Journal of the National Cancer Institute, vol. 82, no. 1, pp. 4-6, 1990.

[85] T. Kaneko, H. Konno, M. Baba, T. Tanaka, and S. Nakamura, "Urokinase-type plasminogen activator expression correlates with tumor angiogenesis and poor outcome in gastric cancer," Cancer Science, vol. 94, no. 1, pp. 43-49, 2003.

[86] C.-N. Chen, F.-J. Hsieh, Y.-M. Cheng et al., "The significance of placenta growth factor in angiogenesis and clinical outcome of human gastric cancer," Cancer Letters, vol. 213, no. 1, pp. 73-82, 2004.

[87] H. Wilke, K. Muro, E. van Custem et al., "Ramucirumab plus paclitaxel versus placebo plus paclitaxel in patients with previously treated advanced gastric or gastro-oesophageal junction adenocarcinoma (RAINBOW): a double-blind, randomised phase 3 trial," The Lancet Oncology, vol. 15, no. 11, pp. 1224-1235, 2014.

[88] H. Li, Y. Adachi, H. Yamamoto et al., "Insulin-like growth factor-I receptor blockade reduces tumor angiogenesis and enhances the effects of bevacizumab for a human gastric cancer cell line, MKN45," Cancer, vol. 117, no. 14, pp. 3135-3147, 2011.

[89] D. Tang, J. Gao, S. Wang et al., "Cancer-associated fibroblasts promote angiogenesis in gastric cancer through galectin-1 expression," Tumor Biology, vol. 37, no. 2, pp. 1889-1899, 2016.

[90] X. Ding, W. Xi, J. Ji et al., "HGF derived from cancerassociated fibroblasts promotes vascularization in gastric cancer via PI3K/AKT and ERK1/2 signaling," Oncology Reports, vol. 40, no. 2, pp. 1185-1195, 2018.

[91] M. B. Meads, R. A. Gatenby, and W. S. Dalton, "Environmentmediated drug resistance: A major contributor to minimal residual disease," Nature Reviews Cancer, vol. 9, no. 9, pp. 665674, 2009.

[92] K. H. T. Paraiso and K. S. M. Smalley, "Fibroblast-mediated drug resistance in cancer," Biochemical Pharmacology, vol. 85, no. 8, pp. 1033-1041, 2013. 
[93] J. Li, J. Guan, X. Long, Y. Wang, and X. Xiang, "Mir-1-mediated paracrine effect of cancer-associated fibroblasts on lung cancer cell proliferation and chemoresistance," Oncology Reports, vol. 35, no. 6, pp. 3523-3531, 2016.

[94] Y. Shintani, A. Fujiwara, T. Kimura et al., "IL-6 secreted from cancer-associated fibroblasts mediates chemoresistance in NSCLC by increasing epithelial-mesenchymal transition signaling," Journal of Thoracic Oncology, vol. 11, no. 9, pp. 14821492, 2016.

[95] F. Lotti, A. M. Jarrar, R. K. Pai et al., "Chemotherapy activates cancer-associated fibroblasts to maintain colorectal cancerinitiating cells by IL-17A," The Journal of Experimental Medicine, vol. 210, no. 13, pp. 2851-2872, 2013.

[96] C.-H. Heldin, K. Rubin, K. Pietras, and A. Östman, "High interstitial fluid pressure-an obstacle in cancer therapy," Nature Reviews Cancer, vol. 4, no. 10, pp. 806-813, 2004.

[97] J. Ma, X. Song, X. Xu, and Y. Mou, "Cancer-associated fibroblasts promote the chemo-resistance in gastric cancer through secreting IL-11 targeting JAK/STAT3/Bcl2 pathway," Cancer Research and Treatment, vol. 51, no. 1, pp. 194-210, 2019.

[98] I. Ham, H. J. Oh, H. Jin et al., "Targeting interleukin-6 as a strategy to overcome stroma-induced resistance to chemotherapy in gastric cancer," Molecular Cancer, vol. 18, no. 1, p. 68, 2019.

[99] K. L. Grogg, C. M. Lohse, V. S. Pankratz, K. C. Halling, and T. C. Smyrk, "Lymphocyte-rich gastric cancer: associations with epstein-barr virus, microsatellite instability, histology, and survival," Modern Pathology, vol. 16, no. 7, pp. 641-651, 2003.

[100] F. Chapel, B. Fabiani, F. Davi et al., "Epstein-Barr virus and gastric carcinoma in Western patients: Comparison of pathological parameters and p53 expression in EBV-positive and negative tumours," Histopathology, vol. 36, no. 3, pp. 252-261, 2000.

[101] M. Wu, C. Shun, C. Wu et al., "Epstein-Barr virus-associated gastric carcinomas: Relation to $\mathrm{H}$. pylori infection and genetic alterations," Gastroenterology, vol. 118, no. 6, pp. 1031-1038, 2000.

[102] A. Aggarwal, "Role of autoantibody testing," Best Practice \& Research: Clinical Rheumatology, vol. 28, no. 6, pp. 907-920, 2014.

[103] A. Poggi, A. Musso, I. Dapino, and M. R. Zocchi, "Mechanisms of tumor escape from immune system: role of mesenchymal stromal cells," Immunology Letters, vol. 159, no. 1-2, pp. 55-72, 2014.

[104] E. Soleymaninejadian, K. Pramanik, and E. Samadian, "Immunomodulatory properties of mesenchymal stem cells: cytokines and factors," American Journal of Reproductive Immunology, vol. 67, no. 1, pp. 1-8, 2012.

[105] A. Abe, A. K. Nagatsuma, Y. Higuchi, Y. Nakamura, K. Yanagihara, and A. Ochiai, "Site-specific fibroblasts regulate site-specific inflammatory niche formation in gastric cancer," Gastric Cancer, vol. 20, no. 1, pp. 92-103, 2017.

[106] E. K. Kim, S. O. Yoon, W. Y. Jung et al., "Implications of NOVA1 suppression within the microenvironment of gastric cancer: association with immune cell dysregulation," Gastric Cancer, vol. 20, no. 3, pp. 438-447, 2017.

[107] S. Yin, Z. Li, J. Huang et al., "Prognostic value and clinicopathological significance of proliferating cell nuclear antigen expression in gastric cancer: A systematic review and metaanalysis," OncoTargets and Therapy, vol. 10, pp. 319-327, 2017.

[108] A. Zhang, Y. Qian, Z. Ye et al., "Cancer-associated fibroblasts promote M2 polarization of macrophages in pancreatic ductal adenocarcinoma," Cancer Medicine, vol. 6, no. 2, pp. 463-470, 2017.
[109] P. Andersson, Y. Yang, K. Hosaka et al., "Molecular mechanisms of IL-33-mediated stromal interactions in cancer metastasis," JCI Insight, vol. 3, no. 20, 2018.

[110] N. Higashino, Y. Koma, M. Hosono et al., "Fibroblast activation protein-positive fibroblasts promote tumor progression through secretion of CCL2 and interleukin- 6 in esophageal squamous cell carcinoma," Laboratory Investigation, 2019.

[111] J.-Y. Blay, L. Shen, Y.-K. Kang et al., "Nilotinib versus imatinib as first-line therapy for patients with unresectable or metastatic gastrointestinal stromal tumours (ENESTg1): A randomised phase 3 trial," The Lancet Oncology, vol. 16, no. 5, pp. 550-560, 2015.

[112] J. Guo, R. D. Carvajal, R. Dummer et al., "Efficacy and safety of nilotinib in patients with kit-mutated metastatic or inoperable melanoma: Final results from the global, single-arm, phase ii team trial," Annals of Oncology, vol. 28, no. 6, pp. 1380-1387, 2017.

[113] A. Hochhaus, G. Rosti, N. C. P. Cross et al., "Frontline nilotinib in patients with chronic myeloid leukemia in chronic phase: results from the European ENEST1st study," Leukemia, vol. 30, no. 1, pp. 57-64, 2016.

[114] E. Day, B. Waters, K. Spiegel et al., "Inhibition of collageninduced discoidin domain receptor 1 and 2 activation by imatinib, nilotinib and dasatinib," European Journal of Pharmacology, vol. 599, no. 1-3, pp. 44-53, 2008.

[115] T. Pang, X. Wang, J. Gao et al., "Fiber-modified hexon-chimeric oncolytic adenovirus targeting cancer associated fibroblasts inhibits tumor growth in gastric carcinoma," Oncotarget, vol. 8, no. 44, pp. 76468-76478, 2017.

[116] Z. Wang, D. Ma, C. Wang et al., "Triptonide inhibits the pathological functions of gastric cancer-associated fibroblasts," Biomedicine \& Pharmacotherapy, vol. 96, pp. 757-767, 2017.

[117] R. Dong, J. Guo, Z. Zhang, Y. Zhou, and Y. Hua, "Polyphyllin I inhibits gastric cancer cell proliferation by downregulating the expression of fibroblast activation protein alpha (FAP) and hepatocyte growth factor (HGF) in cancer-associated fibroblasts," Biochemical and Biophysical Research Communications, vol. 497, no. 4, pp. 1129-1134, 2018.

[118] G. Chen, C. Yu, Z. Tang et al., "Metformin suppresses gastric cancer progression through calmodulinlike protein 3 secreted from tumorassociated fibroblasts," Oncology Reports, vol. 41, no. 1, pp. 405-414, 2019.

[119] I. Tirosh, B. Izar, S. M. Prakadan et al., "Dissecting the multicellular ecosystem of metastatic melanoma by single-cell RNA-seq," Science, vol. 352, no. 6282, pp. 189-196, 2016.

[120] D. Lambrechts, E. Wauters, B. Boeckx et al., "Phenotype molding of stromal cells in the lung tumor microenvironment," Nature Medicine, vol. 24, no. 8, pp. 1277-1289, 2018.

[121] C. Neuzillet, A. Tijeras-Raballand, C. Ragulan et al., "Inter- and intra-tumoural heterogeneity in cancer-associated fibroblasts of human pancreatic ductal adenocarcinoma," The Journal of Pathology, vol. 248, no. 1, pp. 51-65, 2019.

[122] H. Li, E. T. Courtois, D. Sengupta et al., "Reference component analysis of single-cell transcriptomes elucidates cellular heterogeneity in human colorectal tumors," Nature Genetics, vol. 49, no. 5, pp. 708-718, 2017. 


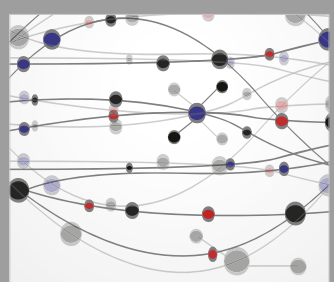

The Scientific World Journal
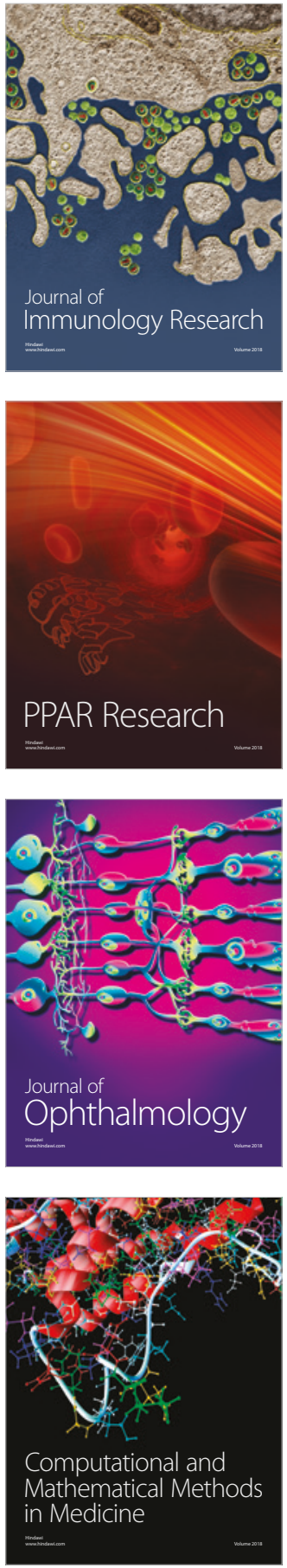

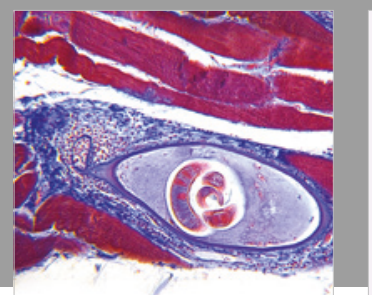

Gastroenterology Research and Practice

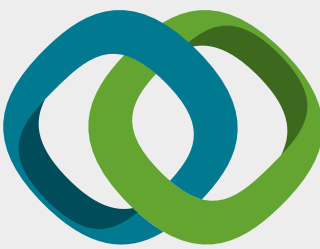

\section{Hindawi}

Submit your manuscripts at

www.hindawi.com
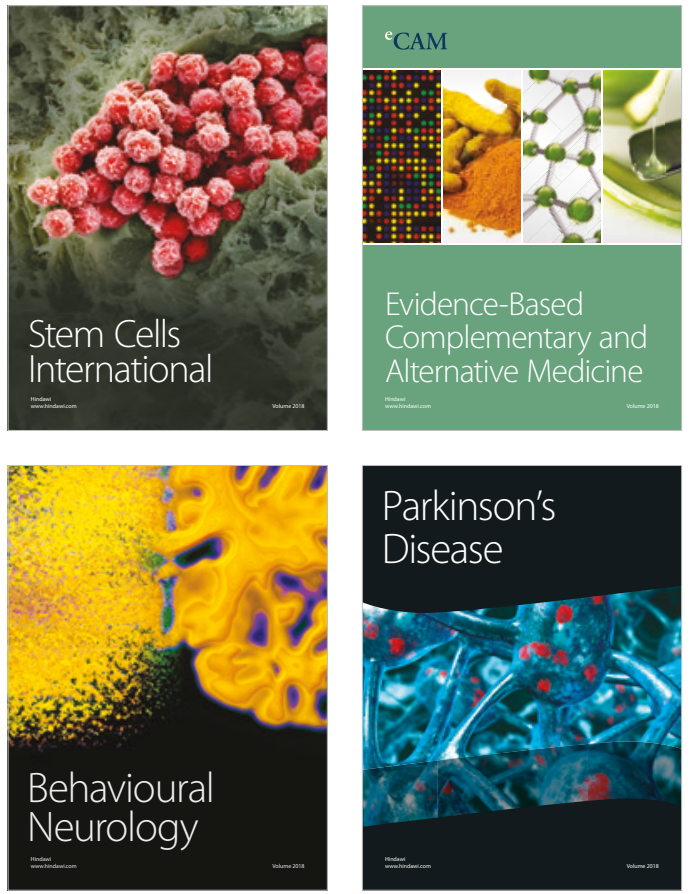

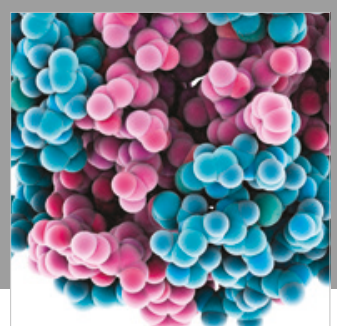

ournal of

Diabetes Research

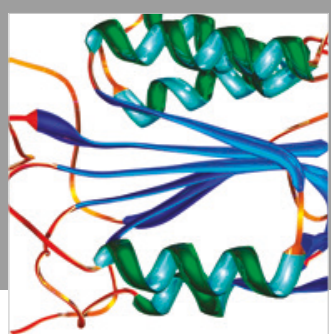

Disease Markers
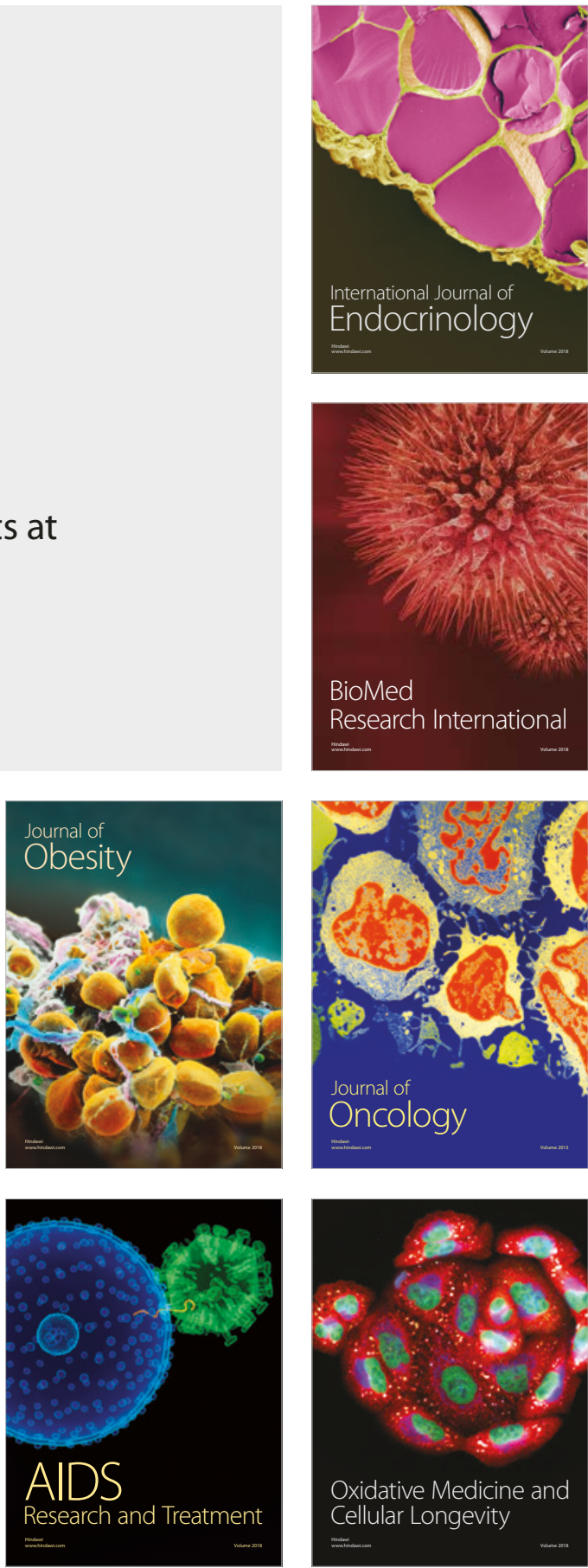